\title{
Evaluation of the efficacy and safety of different class III anti-arrhythmic drugs for treating patients with atrial fibrillation: a network meta-analysis
}

\section{Chuang Yang}

Jilin University Second Hospital

Zhibo Li

Jilin University Second Hospital

\section{Xin Xue}

jilin university second hospital

yu fei ( $\square$ feiyu@jlu.edu.cn )

Jilin University Second Hospital https://orcid.org/0000-0001-7142-8846

Research article

Keywords: Atrial fibrillation, Antiarrhythmic drugs, Network meta-analysis

Posted Date: December 2nd, 2019

DOI: https://doi.org/10.21203/rs.2.17803/v1

License: (1) This work is licensed under a Creative Commons Attribution 4.0 International License.

Read Full License 


\section{Abstract}

Background Class III anti-arrhythmic drugs (AADs) are used to control heart rhythm or rate in the management of atrial fibrillation (AF). In this study, we evaluated the most effective and safe class III AADs used to treat patients with $\mathrm{AF}$.

Methods We searched the PubMed, Cochrane Library, Web of Science, Embase, WanFang databases, as well as the China National Knowledge Infrastructure from 2014 to 2019 to identify studies that reported the use of class III ADDs to treat AF.

Results We identified 31 articles (24 Chinese and 7 English), that included 2,894 AF patients (treatment group $=1,498$, control group $=1,396$ ) for our analysis. Several class III AADs showed significant treatment effects in AF patients compared to conventional treatment. The ADDs were ranked in terms of efficacy from good to bad as follows: vernakalant (relative risk $(\mathrm{RR})=2.7,95 \%$ confidence interval $(\mathrm{Cl}): 1.9$ 3.7), ibutilide ( $R R=2.0,95 \% \mathrm{Cl}: 1.7-2.4$ ), nifekalant ( $R R=1.5,95 \% \mathrm{Cl}: 1.2-2.0)$, and amiodarone ( $R R=1.4,95 \%$ $\mathrm{Cl}$ 1.3-1.5). There were no significant differences in the incidence of adverse reactions among the different class III AADs. These ADDs were ranked from least to most adverse reactions as follows: vernakalant( $R R=0.46,95 \% \mathrm{Cl}: 0.20-1.0)$, sotalol ( $R R=0.52,95 \% \mathrm{Cl}: 0.25-1.0$ ), ibutilide ( $R R=0.64,95 \% \mathrm{Cl}: 0.33-$ 1.2), amiodarone ( $R R=0.79,95 \% \mathrm{Cl}: 0.58-1.1)$, and nifekalant ( $R R=1.0,95 \% \mathrm{Cl}: 0.44-2.5)$.

Conclusion Our network meta-analysis shows that different class III AADs can be used to treat patients with AF both safely and effectively. Vernakalant appears to offer optimal therapeutic effect with minimal adverse reactions.

\section{Background}

Atrial fibrillation (AF) is a type of arrhythmia that can seriously endanger physical and mental health. Epidemiological studies of AF indicate that disability and mortality rates increase every year globally. In recent decades, AF has been reported as a common public health issue [1]. It is thus important to identify optimal treatment strategies for AF patients.

AF can be managed with anti-arrhythmic drugs (AADs) either by controlling heart rhythm or rate [1]. AADs are categorized into four classes based on the Vaughn Williams classification: Class I, sodium channel blockers; Class II, beta receptor blockers; Class III, potassium channel blockers; and Class IV, calcium channel blockers. Previous studies reported that class III AADs help control heart rhythm and rate in AF patients with a low incidence of adverse events compared to class IA AADs [2].Therefore, class III AADs are the most commonly used drugs in the clinical treatment of patients with AF. Previously, class III AADs predominantly included amiodarone and sotalol, but dofetilide, ibutilide, dronedarone, and vernakalant have recently been considered better class III AADs for treating AF. However, clinicians find it difficult to choose the right drug for AF treatment due to differences in effectiveness [3] and safety of class III AADs in the clinic. Importantly, clinicians currently prescribe class III AADs based on either the results of a simple clinical trial that does not reflect the "real world" clinical practice [4-34], or personal preferences 
due to a lack of experimental evidence. In this network meta-analysis we evaluated different class III AADs to determine which drug is most effective and safe for clinical treatment of AF.

\section{Methods}

\section{Materials}

We searched the PubMed, Cochrane Library, Web of Science, Embase, and WanFang databases, as well as the China National Knowledge Infrastructure, to identify studies involving different class III AADs from 2014 to 2019. The main English search terms included: azimilide, ambasilide, amiodarone, dofetilide, dronedarone, ibutilide, sotalol, vernakalant, and nifekalant or atrial fibrillation, respectively. Chinese search terms included:aqilite, anbalite, andiantong, duofeilite, juenaidalong, yibulite, suotaluoer, weinakalan, and nifeikalan or fangchan, respectively.

Take PubMed as an example:

("amiodarone"[MeSH Terms] OR "amiodarone"[All Fields]) AND ("atrial fibrillation"[MeSH Terms] OR ("atrial"[All Fields] AND "fibrillation"[All Fields]) OR "atrial fibrillation"[All Fields]) AND 2014-2019[All Fields]

The inclusion criteria were as follows: 1 ) adult patients with documented AF;2) class III AAD prescribed to treat AF including both intravenous injection and oral administration; 3 ) control group that included cedilanid, placebo, and class III AADs; and 4) electrocardiogram (ECG) results and clinical symptoms were the parameters used to evaluate the indicators. Efficacy of rhythm/rate control and incidence of adverse drug reactions were analyzed from five randomized controlled trials (RCTs).

The exclusion criteria included: 1) basic experimental studies that included animal or cell experiments; 2 ) literature reporting on class III AADs along with other drugs to treat AF, including statins, angiotensin converting enzyme inhibitors (ACEI), ACEI receptor antagonists, or traditional Chinese medicine, published in the title;3)Articles on class III AADs along with other class AADs; 4) class III AAD treatment offered to AF patients who had myocardial infarction and heart failure; 5) repeated publications and undesirable literature; and 5)case reports, dissertations and literature reviews, and incomplete data studies.

\section{Extraction and quality assessment}

We extracted data independently and assessed study quality. Study selection was cross-checked by two reviewers. Controversies in assessments were resolved either by discussion or with the help of third-party reviewer. Study evaluation was conducted following the guidelines provided in the 4.2 Cochrane handbook for systematic reviews of interventions [35] as follows: 1) random allocation method; 2) concealment of allocation; 3) blinding method; 4) integrity of the resulting data; 5) selective reporting of the results; and 6 ) other sources of bias. Based on these criteria, the studies were graded as follows: $A$ : meets all 6 criteria and hence is without much bias; B: 1 or 2 criteria are partially satisfied and hence the 
study may be moderately biased; C: 1 or more criteria might completely disagree and hence the study is considered to be biased.

\section{Statistical methods}

Dichotomous data were analyzed and expressed asrelative risk (RR). The 95\% confidence intervals (Cl) were also calculated; a $95 \% \mathrm{Cl}$ that included or contacted 1 was equivalent to $\mathrm{P}>0.05$, while a $95 \% \mathrm{Cl}$ that excluded 1 was equivalent to $P<0.05$. Statistical analysis of heterogeneity was presented and considered significant when the I square $\left(\mathrm{I}^{2}\right)$ value exceeded $50 \%$ or $\mathrm{P}<0.05$. The random-effects model was used as a follow-up analysis. Data pooling witha fixed-effects model was used in the case where statistical heterogeneity was $\left.\right|^{2}<50 \%$ or $P>0.05$. A funnel plot was used to analyze the risk of bias and the network plot was used to represent the relationship between different studies. The connecting lines and no-lines indicated the evidence of direct and indirect comparisons between two interventions; the thickness of the line indicated the number of direct comparisons included, while the point size indicated the total number of samples. Consistent and inconsistent models were determined by $P>0.05$ and $P<0.05$, respectively. The inconsistency factor (IF) was used to express the consistency test. The trajectory density graph determined by observation Markov chain-Monte Carlo (MCMC) and bandwidth value were used to ensure the convergence degree of the model. The merits and demerits of various measures were assessed through the ranking probability plot and forest plot following comparison between multiple interventions. We used Endnote X9 software for document processing. Stata 15 software was used to generate forest plots, network plots, and consistent models plots. Finally, we used R software (version 3.4.4) for ranking plot, trajectory density graph, and heterogeneity detection by calling for the GeMTC package and Just another Gibbs sampler (JAGS) software. Statistical differences determined at $\mathrm{P}<0.05$ were considered significant.

\section{Results}

\section{Retrieval strategy}

We retrieved 6,389 studies but eliminated 3,596 duplicates. We further excluded 2,607 studies because they were not considered relevant. We read the full-text and abstracts of the remaining 186 studies, and identified 31 eligible articles [4-34]. Among these, 24 were in Chinese [4-27] and the remaining 7 were in English [28-34] (Fig. 1).

\section{Document characterization}

The included studies involved a total of 2,894 patients for analysis, and among them 1,498 belonged to the treatment group and the remaining 1,396 belonged to the control group. We studied the efficacy of six class III AADs, including amiodarone, dronedarone, ibutilide, nifekalant, sotalol, and vernakalant. We studied the adverse effects of five class III AADs, including amiodarone, ibutilide, nifekalant, sotalol and vernakalant. All analyses were carried out using bi-arm studies. The literature refers to paroxysmal, 
persistent, and permanent $A F$, as reported in the references provided here: paroxyxmal $A F[4,7,9,10,13$, $14,18,20,22,24,31,32]$, persistent AF $[5-7,15,16,22,25,26,28]$, and permanent AF [7]. Duration of AF was explained in $[4,6,8,14-16,18,22,23,26-30,32-34]$, and was referred to as AF pathogeny as follows: valvular heart disease $[5,8,10,12,17,20,22,23,26,29,34]$, hypertensive heart disease $[10,12$, $20,22,25,26,28,30,32-34]$, coronary heart disease [10, 12, 20, 22, 25, 26, 28-30, 32-34], and dilated cardiomyopathy $[12,28]$. The general quality grade of the studies was $B$ [28-32]; in a few exceptions the grade was C [4-27, 33, 34] (Table.1).

\section{The treatment effect of several class III AADs for patients with AF}

Efficiency rates were reported in 31 articles and they included two closed rings, namely amiodaroneibutilide-nifekalant $(\mathrm{IF}=0.481, \mathrm{P}=0.086)$ and amiodarone-conventional treatment-sotalol $(\mathrm{IF}=0.186, \mathrm{P}=$ 0.241), for which consistent models were used. Fixed models were applied using $\mathrm{I}^{2}=36.71 \%$.

The relationship between amiodarone, dronedarone, ibutilide, nifekalant, sotalol, and vernakalant and conventionally treated AF patients were presented using the network plot. Amiodarone use was reported in a majority of the samples, followed by conventional treatment and ibutilide. Direct comparison showed a high relationship between amiodarone and conventional treatments, and amiodarone and ibutilide with the second highest relationship (Fig. 2).

We set the iteration to 10,000 times, and showed that the MCMC, as well as the density map convergence, were better in the iterative operation of 40,000 times. The treatment effect of four interventions was significantly increased [amiodarone $(R R=1.4,95 \% \mathrm{Cl}: 1.3-1.5)$, ibutilide $(\mathrm{RR}=2.0,95 \% \mathrm{Cl}: 1.7-2.4)$, nifekalant $(\mathrm{RR}=1.5,95 \% \mathrm{Cl}: 1.2-2.0)$, and vernakalant $(\mathrm{RR}=2.7,95 \% \mathrm{Cl}: 1.9-3.7)]$ compared to the conventional treatment. However, two interventions [dronedarone $(\mathrm{RR}=1.0,95 \% \mathrm{Cl}: 0.91-1.1)$ and sotalol ( $R R=1.0,95 \% \mathrm{Cl}: 0.91-1.1)]$ showed no significant differences. We arranged all six interventions in the following order based on efficacy: vernakalant, ibutilide, nifekalant, amiodarone, dronedarone, and sotalol (Fig. 3).

Incidence of adverse events of several class III AADs in patients with AF

Incidence of adverse reactions was reported in 20 articles. There were two closed ring [amiodaroneibutilide-nifekalant ( $\mathrm{IF}=0.549, \mathrm{P}=0.712)$ and amiodarone-conventional treatment-sotalol $(\mathrm{IF}=0.081, \mathrm{P}=$ 0.926)],models consistently used in the articles. Fixed models were applied using $\mathrm{I}^{2}=0 \%$.

The relationship between amiodarone, ibutilide, nifekalant, sotalol, and vernakalant and conventional treatment of patients with AF was determined using the network plot. Amiodarone was present in a majority of the samples, followed by conventional treatment and ibutilide. Direct comparison showed amiodarone and conventional treatments had a higher relationship, following by amiodarone and ibutilide (Fig. 4). 
We had set the iteration to 10,000 times and showed that the MCMC, as well as the density map convergence, were better in the iterative operation of 40,000 times. Incidence of adverse reactions of five interventions showed no significant difference in conventional treatment [amiodarone $(R R=0.79,95 \%$ $\mathrm{Cl}: 0.58-1.1)$, ibutilide $(\mathrm{RR}=0.64,95 \% \mathrm{Cl}: 0.33-1.2)$, nifekalant $(\mathrm{RR}=1.0,95 \% \mathrm{Cl}: 0.44-2.5)$, sotalol $(\mathrm{RR}=$ $0.52,95 \% \mathrm{Cl}: 0.25-1.0)$, and vernakalant $(\mathrm{RR}=0.46,95 \% \mathrm{Cl}: 0.20-1.0)]$. The ADDs were arranged based on the incidence of adverse reaction from least to most as follows: vernakalant, sotalol, ibutilide, amiodarone, and nifekalant (Fig. 5).

The risk of bias in several class III AADs for patients with AF

A funnel plot was used to indicate the bias risk of the treatment effect and incidence of adverse events. Analysis showed symmetry of both limbs, with most samples distributed at the top, indicating no significant bias risk (Figs. 6 and 7).

\section{Discussion}

The purpose of this network meta-analysis was to analyze the safety and efficacy of different class III AADs in the treatment of AF. The results showed that several ADDs have obvious therapeutic effects with minimal incidence of adverse events. We found that vernakalant had the best therapeutic effect with the least incidence of adverse reactions.

$\mathrm{AF}$ is a type of atrial tachyarrhythmia that is characterized by rapid and disordered atrial electrical activity. These irregularities shorten ventricular diastole and further reduce cardiac output, leading to a decrease in blood pressure and coronary perfusion. This induces or aggravates myocardial ischemia and leads to tachycardia cardiomyopathy. Heart rhythm and rate control can alleviate AF symptoms and improve prognosis [1]. Class III AADs have been shown to be an effective clinical strategy for treating patients with AF. Class III ADDs not only control heart rhythm or rate, but also significantly reduce the occurrence of adverse events [2,4-34]. Previous studies that investigated the efficacy of class III AADs in the treatment of AF patients were limited to direct comparisons only. While the results obtained in those studies may be accurate and reliable, the interventions were one-sided without indirect comparison. We addressed this limitation by conducting a network meta-analysis. We retrieved articles reporting RCTs from PubMed and other databases that discussed the role of class III AADs in treating AF patients between 2014 and 2019. The chief six ADD interventions obtained from 31 articles included: $80.6 \%$ amiodarone (25), 6.4\% dronedarone (2), 38.7\% ibutilide (12), 6.4\% nifekalant (2), 6.4\% sotalol (2), and $6.4 \%$ vernakalant (2). Our network meta-analysis showed the efficacy of different class III AADs in treating patients with AF. Among them, amiodarone, ibutilide, nifekalant, and vernakalant were considered to be effective, and amiodarone, ibutilide, nifekalant, sotalol, and vernakalant were found to be safe.

Dronedarone has a similar chemical structure to amiodarone and is thus referred to as a secondgeneration amiodarone. Further research conducted by Guerra et al., confirmed that dronedarone is the most effective AAD for treating AF and is even tolerated by patients who previously presented with an inability to tolerate other AADs [29]. According to Ezekowitz et al., dronedarone can prevent the 
occurrence of AF [30]. However, there are studies that indicate that dronedarone treatment in patients with AF is less effective compared to amiodarone[36,37]. From our results we are unable to conclude if dronedarone is superior to conventional treatment for managing AF. This may be due to a lack of relevant literature and research methods.

Previous studies showed that the treatment efficacy and incidence of adverse reactions differed in patients with AF who were administered class III AADs. For example, Ding et al., reported that ibutilide was more effective compared to amiodarone, but the incidence of adverse reactions did not differ statistically [15]. Dai et al., found no significance differences in treatment effect between nifekalant and amiodarone, but did report that nifekalant improved AF clinical symptoms more quickly compared to amiodarone [24]. Qin et al., showed that both amiodarone and sotalol were more effective compared to dronedarone for controlling heart rhythm [3]. Kriz et al., reported that there was no significant difference in the efficacy of ibutilide, vernakalant, and amiodarone [38]. However, it is important to note that none of these studies confirmed which of the class III ADDs was the most efficient and safest for treating AF. Our results showed that vernakalant had the best therapeutic effects, followed by ibutilide and nifekalant; vernakalant also resulted in the least incidence of adverse reactions, followed by sotalol and ibutilide. In our analysis of six class III AADs, we did not find any data on the adverse reactions of dronedarone. Previous studies showed that the occurrence of adverse reactions in dronedarone were similar compared to amiodarone, with a decreased incidence of thyroid toxicity, lung toxicity, and ophthalmia [36].Thus, the incidence of adverse reactions of dronedarone are not better compared to vernakalant, although there was a lack of sorting probability for the dronedarone data. Based on the above findings, we conclude that vernakalant is the best class III AAD for treating AF.

The efficacy and occurrence of adverse reactions of different class III AADs were determined by the effect of the drug on different potassium channel subtypes, blocking other ion channels, and myocardial site of drug selective action. The main ion current during myocardial cell repolarization is potassium ion outflow, which includes fast activation of delayed rectification current (Ikr), slow activation of delayed rectification current (Iks), ultrafast delayed rectification current (Ikur), transient outward potassium current (Ito), acetylcholine-sensitive potassium current (IkAch), and inward rectifier potassium current (IKir) [39]. It has been found that blocking IKr will lead to negative frequency-dependent regulation of action potential duration (APD), resulting in slower heart rate due to prolonged APD. Excessive prolongation of APD induces early-posterior potential and tip torsional ventricular tachycardia, which increases the risk of death. Its representative drugs include sotalol, nifekalant, and ibutilide. In contrast, blocking IKs will lead to positive frequency-dependent regulation of APD. APD and effective refractory period (ERP) are significantly prolonged with increases in heart rate. At the same time, decreases in heart rate will prolong the APD and ERP to a lower rate. Thus, the pro-arrhythmogenic effect of these drugs is weaker compared to the IKr blocker. Its representative compounds include HMR-1556 and Chromanol 293B. Blocking IKr and IKs represent amiodarone, dronedarone, respectively [40, 41]. Apart from the above-mentioned potassium currents, Ikur is the chiefly formed potassium current and is formed by vernakalant. Ikur is the main current during APD repolarization in cardiomyocytes [42].Vernakalant acts on Kv1.5 ion channels and has high selectivity in the atrium. It also only acts on human atrial myocytes, not ventricular 
myocytes [43]. Finally, vernakalant blocks Ito, IkAch, delayed sodium channel current (INa), and other multi-ion channels [39]. Therefore, there is justification that vernakalant has the best therapeutic effect and safety among other AADs.

The plot in our study was symmetrical and roughly funnel-form in shape, but does not allow us to exclude all potential biases for the following reasons: 1 ) We could not elaborate on all diseases that cause AF mentioned in the included 31 articles. For instance valvular heart disease $[5,8,10,12,17,20,22,23,26$, $29,34]$, hypertensive heart disease [10, 12, 20, 22, 25, 26, 28, 30, 32-34], coronary heart disease [10, 12 , $20,22,25,26,28-30,32-34]$, and dilated cardiomyopathy [12, 28]were treated differently. More specifically, coronary heart disease was treated with lipid-lowering and antiplatelet drugs, and hypertension was treated with pressure reducing drugs.2) AF classification was randomly mixed in the included 31 articles, and the literature included a collection of paroxysmal $\operatorname{AF}[4,7,9,10,13,14,18,20$, $22,24,31,32]$, persistent AF $[5-7,15,16,22,25,26,28]$, and permanent AF[7]. The literature also included duration of $\operatorname{AF}[4,6,8,14-16,18,22,23,26-30,32-34]$,treatment course, efficacy judgment (conversation rate, effective rate), and drug administration. In addition, the sample size, quality of the selected documents, unknown languages, and geographical location bias may have influenced heterogeneity of the included studies. Due to these shortcomings in our meta-analysis, we recommend further detailed research with large sample evidence-based tests and a multicenter study.

\section{Conclusion}

Our network meta-analysis indicates that different class III AADs can effectively and safely treat patients with AF. Our analysis also indicates that vernakalant provides optimal therapeutic effects and has the least incidence of adverse reactions. Therefore, vernakalant should be considered for clinical application to treat AF patients.

\section{Abbreviations}

\section{AADs}

anti-arrhythmic drugs; AF:atrial fibrillation; ECG:electrocardiogram; RCTs randomized controlled trials;. ACEI angiotensin converting enzyme inhibitors; RR asrelative risk; $\mathrm{Cl}$ Confidence interval; IF:inconsistency factor; MCMC:Markov chain-Monte Carlo.

\section{Declarations}

Competing interests:

The authors declare that there are no competing interests

Funding:

This work was not source of financial support in the form of grants; 
Authors' contributions:

Conception and design of the research: $\mathrm{CY}, \mathrm{XX}, \mathrm{YF}$.

Acknowledgements:

None

Authors' information (optional)

Department of Cardiology, The Second Hospital, Jilin University, Changchun, Jilin 130041, China

\section{References}

1.

Khaji A, Kowey PR. Update on atrial fibrillation. Trends Cardiovasc Med. 2017;27:14-25.

2.

Lafuente-Lafuente C, Valembois L, Bergmann JF, Belmin J. Antiarrhythmics for maintaining sinus rhythm after cardioversion of atrial fibrillation. Cochrane Database Syst Rev. 2015;28(3):CD005049.

3.

Qin D, Leef G, Alam MB, Rattan R, Munir MB, Patel D, et al. Comparative effectiveness of antiarrhythmic drugs for rhythm control of atrial fibrillation. J Cardiol. 2016;67:471-6.

4.

Li Y. Comparison of the clinical efficacy between amiodarone and cedilanid in the treatment of rapid paroxysmal atrial fibrillation with intravenous administration. China Foreign Medical Treatment. 2014;20:8-9.

5.

Xuan HY, Shi KH, Xu SS, Zhao XD, Zhan HY, Wang YQ. Clinical study of immediate cardioversion of persistent atrial fibrillation after failed intraoperative radiofrequency ablation with ibutilide versus amiodarone. Acta UniversitatisMedicinalis Anhui. 2014;49:127-9.

6.

XiaoYQ.Comparative study of. efficacy and safety between ibutilide and amiodarone for cardioversion of persistent atrial fibrillation to sinus rhythm. Chin J Cardiovascular Research. 2014;12:837-40.

7.

Su XM. Observation on the efficacy and side effects of amiodarone in the treatment of atrial fibrillation. $J$ Medical Theory Practice. 2014;27:321-2.

8.

HuangY. Evaluation of amiodarone in the treatment of atrial fibrillation after valve replacement in elderly patients with rheumatic heart disease. Contemporary Medicine. 2014;20:28-9.

9.

Zhou YY, Lu GL, Wu M. Clinical effect of amiodarone and trichosanthin C on paroxysmal atrial fibrillation and atrial flutter. Chin J Gerontology. 2014;34:64-5.

10 .

Wu LN. Clinical evaluation of amiodarone and cedilanid in the treatment of paroxysmal atrial fibrillation. Chin Foreign Med Research. 2015;13:140-1. 
11.

Wu L, Qu H, Liu HT, Zhang HZ, Li XM. Clinical observation and nursing of amiodarone in the treatment of atrial fibrillation. Chin J Trauma Disability Med. 2015;23:174-5.

12.

Zhao XM, Jiang HM. Clinical observation of amiodarone in the treatment of 38 cases of rapid atrial fibrillation. World Latest Medicine Information. 2015;15:90-1.

13.

Du L, Wang GT, Liu JH. Clinical effect of amiodarone on paroxysmal atrial fibrillation. Medical Information. 2015;28:156-7.

14.

Li Yue, Zhou J. Clinical observation of 42 cases of amiodarone cardioerter paroxysmal atrial fibrillation. Modern Chinese Doctor. 2015;53:21-3.

15.

Ding Y, Sun, JG,Wu. JR,Zhang XP.Clinical efficacy of amiodarone and ibutilide on persistent atrial fibrillation in elderly patients after ablation. China J ClinPharmacol. 2016;32:570-2.

16.

Zhao YJ, Yan L. Amiodarone and sotalol after radiofrequency ablation of persistent atrial fibrillation control study.ChinaPrac. Med. 2016;11:159-60.

17.

Hong W. Jiang Q.Evaluation of amiodarone in the treatment of atrial fibrillation after valve replacement in elderly patients with rheumatic heart disease. Medical Forum. 2016;20:4684-5.

18.

Hu M, Ge HS, Li Y, Fei JL. Clinical efficacy of intravenous amiodarone in the treatment of paroxysmal atrial fibrillation. J Clinical Med. 2016;3:7309-10.

19.

Lu QH. Effectiveness of amiodarone in the treatment of rapid atrial fibrillation in patients with rheumatic heart disease. For All Herlth. 2016;10:164.

20.

Zhou CW. Clinical efficacy of intravenous injection of amiodarone and cedilanid in the treatment of rapid atrial fibrillation. China Medicine Pharmacy. 2017;7:169-71.

21.

Su YA, Pan ZY, Wei RL. Comparison of clinical effects of amiodarone and cedilanid on ventricular rate in patients with rapid atrial fibrillation. J Electrocardiogram(Electronic Edition). 2017;6:178-9.

22.

Sun L, Zhao HW. ZhangWW.Comparison of efficacy and safety analysis of ibutilide and amiodarone in cardioversion of atrial fibrillation. Guide of ChinaMedicine. 2018;16:49-50.

23.

Zhao GX, Wang Y. LiuZQ.Effect of amiodarone on rheumatic heart disease with atrial fibrillation influencing factors of prognosis. J Guizhou Med. 2018;42:1433-4.

24. 
Dai Y, Xie HX, Kan JY,Zhou P. Comparison of the efficacy of intravenous nifekalant and amiodarone in treatment of patients with paroxysmal atrial fibrillation.South China. J Cardiovascular Dis. 2019;25:1924.

25.

Cui HM. Tang YH,Wan WT,Zhang JY,Liao DN.Clinical efficacy of nifekalant and ibutilide for cardioversion of persistent atrial fibrillation after radiofrequency catheter ablation. Chin J Cardiac Arrhythmias. 2019;23:39-42.

26.

Li JQ, Shen HM,Guo L, Yang GB,Yuan. JQ.Effects of amiodarone and ibutilide sequential treatment on efficacy and transmural dispersion of repolarization in cardioversion of new persistent atrial fibrillation. Chin J Arterioscler. 2019;27:426-31.

27.

Fu FX. Wang YH.Clinical observation of amiodarone in the treatment of atrial fibrillation. Electronic $\mathrm{J}$ General Stomatology. 2019;6:41-2.

28.

Galperin J, Elizari MV, Bonato R, Ledesma R, Vazquez Blanco M, Lago M, et al. Short-term amiodarone therapy after reversion of persistent atrial fibrillation reduces recurrences at 18 months. Cardiol J. 2014;21:397-404.

29.

Guerra F, Hohnloser SH, Kowey PR, Crijns HJ, Aliot EM, Radzik D, et al. Efficacy and safety of dronedarone in patients previously treated with other antiarrhythmic agents. Clin Cardiol. 2014;37:717-24. 30.

Ezekowitz MD, Ellenbogen KA, DiMarco JP, Kaszala K, Boddy A, Geba GP, et al. A placebo-controlled, double-blind, randomized, multicenter study to assess the effects of dronedarone $400 \mathrm{mg}$ twice daily for 12 weeks on atrial fibrillation burden in subjects with permanent pacemakers. J Interv Card Electrophysiol.2015;42:69-76. [31]Meng ZW, Tan J, He Q, Zhu M, Li X, Zhang JP, et al. Wenxin Keli versus sotalol for paroxysmal atrial fibrillation caused by hyperthyroidism: a prospective, open label, and randomized study. Evid Based Complement Alternat Med. 2015; doi: 10.1155/ 2015/101904. 32.

Vilvanathan VK, Srinivas Prabhavathi Bhat BC, Nanjappa MC, Pandian B, Bagi V, Kasturi S, et al. A randomized placebo-controlled trial with amiodarone for persistent atrial fibrillation in rheumatic mitral stenosis after successful balloon mitral valvuloplasty. Indian Heart J. 2016;68:671-7.

33.

Simon A, Niederdoeckl J, Skyllouriotis E, Schuetz N, Herkner H, Weiser C, et al. Vernakalant is superior to ibutilide for achieving sinus rhythm in patients with recent-onset atrial fibrillation: a randomized controlled trial at the emergency department. Europace. 2017;19:233-40.

34.

Vogiatzis I, Papavasiliou E, Dapcevitch I, Pittas S, Koulouris E. Vernakalant versus ibutilide for immediate conversion of recent-onset atrial fibrillation. Hippokratia. 2017;21:67-73.

35 .

Page $11 / 12$ 
Liu M. System evaluation, meta-analysis design and implementation method. 1th ed. Beijing: People's Medical Publishing House; 2011. p. 66. Chap. 6.

36.

Lei ZB. Adverse reactions and interactions of the antiarrhythmic drug dronedarone. Chin J New Drugs. 2012;21:1826-9.

37.

Køber L, Torp-Pedersen C, McMurray JJ, Gøtzsche O, Lévy S, Crijns H, et al. Dronedarone study group. increased mortality after dronedarone therapy for severe heart failure. N Engl J Med. 2008;358:2678-87. 38.

Kriz R, Freynhofer MK, Weiss TW, Egger F, Gruber SC, Eisenburger P, et al. Safety and efficacy of pharmacological cardioversion of recent-onset atrial fibrillation: a single-center experience. Am J Emerg Med. 2016;34:1486-90.

39.

Zhou Y, Wang J, Wang MH. Application of vernakalant in the treatment of atrial fibrillation. World Clin Drugs. 2015;36:68-71.

40.

Kofune T, Watanabe I, Okubo K, Okumura Y, Masaki R, Shindo A, et al. Effect of IKr blocker nifekalant on atrial action potential duration after successful internal cardioversion of chronic atrial fibrillation.PacingClinElectrophysiol.2005; 28: 391-6.

41.

Huang CX. Advances in Class III antiarrhythmic drugs. Chin J Practical Internal Med. 2006;26:1279-80. 42.

Zhang BY, Li GP. Application of new antiarrhythmic drugs in atrial fibrillation. J Tianjin Medical University. 2017;23:90-2.

43.

Gong YZ, Liu XX, Wu J, Ding WG, Li XQ. Development in atrial fibrillation and Kv1.5 potassium channel blockers research.Progress in Modern Biomedicine. 2015; 15:2386-9. 